\title{
Long Noncoding RNA Cancer Susceptibility Candidate 9 Promotes Chemotherapy Resistance of Non-Small Cell Lung Cancer
}

\author{
DING ZHU CHEN*, YI ZHANG, YONG LIN, YU ZHEN HUANG ${ }^{1}$, ZHI JIAN ZHANG ${ }^{2}$, JI GUANG ZHOU ${ }^{3}$, QING YONG TIAN \\ AND JUN BIAO CHEN
}

Department of Cardiac and Thoracic, ${ }^{1}$ Department of pathology, ${ }^{2}$ Department of Clinical Laboratory, ${ }^{3}$ Department of Information Center, Zhangzhou Hospital Affiliated to Fujian Medical University, No. 59, Shenglixi Road, Zhangzhou, Fujian 363000,

P. R. China

\section{Chen et al.: Chemotherapy Resistance of Non-Small Cell Lung Cancer}

To investigate the molecular mechanism of long noncoding RNA cancer susceptibility candidate 9 involved in gefitinib resistance in non-small cell lung cancer cells by regulating microRNA-382-5p/phosphoinositidedependent protein kinase-1/mammalian target of rapamycin complex 2 signal axis. 10 cases of serum before treatment and 10 cases of drug resistant serum after gefitinib treatment were collected from December 2019 to December 2020. The expression levels in non-small cell lung cancer tissues or cell lines were detected by real-time quantitative reverse transcription polymerase chain reaction. Cell counting kit 8, transwell and cell flow cytometry were used to detect the sensitivity of non-small cell lung cancer cells to gifetinib. Double luciferase reporter assay was used to verify the targeting relationship and the regulatory relationship was detected by western blotting and real-time quantitative reverse transcription polymerase chain reaction. Cancer susceptibility candidate 9 was highly expressed in non-small cell lung cancer tissues and drug-resistant $\mathrm{HCC827/G}$ cells. Overexpression of cancer susceptibility candidate 9 can significantly promote the proliferation, invasion and inhibit apoptosis of HCC827/G cells. At the same time, dual luciferase reporter assay confirmed that cancer susceptibility candidate 9 could negatively regulate the expression of microRNA-382-5p and microRNA-185-5p could bind to the 3'UTR of phosphoinositidedependent protein kinase-1 and mammalian target of rapamycin complex 2 and negatively regulate the expression of phosphoinositide-dependent protein kinase-1 and mammalian target of rapamycin complex 2. Further experiments showed that cancer susceptibility candidate 9 could up regulate the drug resistance of $\mathrm{HCC827/G}$ cells to gefitinib by down regulating the inhibition of microRNA-382-5p on phosphoinositide-dependent protein kinase-1 and mammalian target of rapamycin complex 2 , thereby promoting the proliferation, invasion and inhibiting apoptosis of $\mathrm{HCC827/G}$ cells. Cancer susceptibility candidate 9 induces gefitinib resistance in non-small cell lung cancer cells by regulating microRNA-3825p/phosphoinositide-dependent protein kinase-1/mammalian target of rapamycin complex 2 signal axis.

Key words: Non-small cell lung cancer, gefitinib, microRNA-382-5p, rapamycin

Lung cancer is one of the most common malignant tumors in clinic and its mortality rate ranks the first $\mathrm{t}^{[1]}$. The incidence rate and mortality rate of lung cancer show a sharp upward trend ${ }^{[2]}$. Lung cancer is mainly divided into non-small cell lung cancer (NSCLC) and small cell lung cancer (SCLC) and NSCLC is the main phenotype. NSCLC has the characteristics of high recurrence rate and easy metastasis. Gefitinib is a common chemotherapy drug in the treatment of NSCLC, but more and more patients show significant drug resistance ${ }^{[3]}$. It is the focus of clinical and basic research to study the mechanism of drug resistance of NSCLC cells and provide more effective chemosensitization methods. Research found that long non coding RNA (lncRNA) was abnormally expressed in drug-resistant and drug sensitive lung cancer cells, which may lead to drug resistance of patients ${ }^{[4]}$. Long noncoding RNA cancer susceptibility candidate 9

*Address for correspondence

E-mail: chendingzhufjmu@sohu.com 
(lncRNA CASC9) was up-regulated in bladder cancer and melanoma tissues and cells, and its expression level was related to the proliferation, migration, invasion and apoptosis of tumor cells ${ }^{[5,6]}$. Lncbase predicted v. 2 predicted that there were complementary binding sites between MicroRNA-382-5p (miR-382-5p) and CASC9. Mir-382-5p was low expressed in NSCLC ${ }^{[7]}$ and overexpression of miR-382-5p can increase the chemosensitivity of pancreatic ductal adenocarcinoma cells to gemcitabine ${ }^{[8]}$. However, the expression of CASC9 and miR-382-5p in HCC827/G, the relationship between them and the mechanism of their effect on drug sensitivity of HCC 827 cells are still unclear. Therefore, this study was to investigate the effects of CASC9 and miR-382-5p on the proliferation, invasion, migration and gefitinib sensitivity of HCC 827 cells and the underlying mechanism, so as to provide a new research direction for the drug resistance of NSCLC.

\section{MATERIALS AND METHODS}

\section{Materials:}

HCC827 cell line was purchased from Shanghai Institute of cells, Chinese Academy of Sciences. HCC $827 / G$ cells were constructed by continuously stimulating HCC827 cells with low concentration of gefitinib. 10 cases of serum before treatment and 10 cases of drug-resistant serum after gefitinib treatment were collected from December 2019 to December 2020, including 12 males and 8 females, aged 49-73 y.

\section{Drugs, reagents and instruments:}

Small interfering RNA (siRNA) and mimics of CASC9, miR-382-5p, phosphoinositide-dependent protein kinase-1 (PDK1) and mammalian target of rapamycin complex 2 (mTORC2) were purchased from Shanghai Gemma Company. Dulbecco's modified eagle medium (DMEM) and fetal bovine serum were purchased from biological industries company. Penicillin and streptomycin were purchased from Beijing Regan Biotechnology Co., Ltd. Annexin V-FITC/PI apoptosis detection kit was purchased from ebioscience company and cell counting kit-8 (CCK-8) kit was purchased from Wuhan Huamei Bioengineering Co., Ltd. Transwell small room was purchased from Corelle, USA, DNA enzyme and Lipofectamine ${ }^{\mathrm{TM}} 2000$ and reverse transcriptase kit were purchased from Japan TaKaRa company, high purity total RNA rapid extraction kit purchased from Beijing hundred Tek Biotechnology Co., Ltd., full protein extraction kit, nuclear protein and cytoplasm protein extraction kit, sodium dodecyl sulfate polyacrylamide gel electrophoresis gel rapid preparation kit were purchased from Bio-Rad company of America. Both western blotting anti-1 and anti-secondary were purchased from CST Company of the United States; dual luciferase reporter gene kit and reporter gene vector were purchased from Promega Company. Enzyme labelling, fluorescence quantitative PCR, electrophoresis and gel imaging systems were purchased from the Thermo Fisher Scientific Company of the United States. The ultraspeed centrifuge and electrophoresis tank were purchased from Beijing 61 Biotechnology Co., Ltd.

\section{Cell culture:}

HCC 827 cells were cultured in the DMEM medium containing $10 \%$ fetal bovine serum, $100 \mathrm{U} / \mathrm{ml}$ penicillin and $100 \mu \mathrm{g} / \mathrm{ml}$ streptomycin in $37^{\circ}, 5 \%$ $\mathrm{CO}_{2}$ incubator.

\section{Establishment of drug resistant NSCLC cell line:}

The HCC 827 cells were treated with gefitinib at a beginning concentration of $0.001 \mu \mathrm{g} / \mathrm{ml}$ for $48 \mathrm{~h}$, then the medium was discarded and DMEM medium was added to continue the culture. The cell viability was observed. If there was no obvious death, the cells were selected to gradually increase the concentration of gefitinib. The cells were subcultured repeatedly until they could survive stably in gefitinib at the concentration of $1 \mu \mathrm{g} / \mathrm{ml}$, which named $\mathrm{HCC} 827 / \mathrm{G}$ cells.

\section{Cell transfection:}

NSCLC HCC827 cells in logarithmic growth phase were digested with trypsin and then the density was adjusted to $1 \times 10^{5} / \mathrm{ml}$ using the DMEM medium. Then, $2 \mathrm{ml}$ cell suspension were seeded on a 6 -well plate and cultured in a $5 \% \mathrm{CO}_{2}$ incubator at $37^{\circ}$ for $24 \mathrm{~h}$. Then CASC9 siRNA and pcDNA-CASC9 were transfected to the cells. The transfection method was according to the instructions of LipofectamineTM 2000 transfection reagent. $48 \mathrm{~h}$ after transfection, the transfection effect was observed under fluorescence microscope. Meanwhile, HCC $827 / \mathrm{G}$ cells in logarithmic growth phase were digested with trypsin and then the density was adjusted to $1 \times 10^{5} / \mathrm{ml}$ using the DMEM medium. Then, $2 \mathrm{ml}$ cell suspension were seeded on a 6 -well plate and cultured in a $5 \% \mathrm{CO}_{2}$ incubator at $37^{\circ}$ for $24 \mathrm{~h}$. Then CASC9 siRNA, si-PDK/mTORC2, pcDNA-CASC9 and miR-382-5p mimics were transfected to the 
cells. The transfection method was according to the instructions of Lipofectamine ${ }^{\mathrm{TM}} 2000$ transfection reagent. $48 \mathrm{~h}$ after transfection, the transfection effect was observed under fluorescence microscope.

\section{Real-time quantitative reverse transcription polymerase chain reaction (qRT-PCR):}

Clinical samples and NSCLC HCC827 and HCC $827 / G$ cells $48 \mathrm{~h}$ after transfection were collected and total RNA was extracted from tissues and cultured cells by Trizol for qRT-PCR. U6 and Glyceraldehyde 3-phosphate dehydrogenase (GAPDH) were used as the control and the primer sequences were shown as follows: PDK1, F: 5'-CTACCAGCCATGTCAGAGGATG-3', R: 5'-AGGCTGGTTTCCACCGTAGACA-3'; mTORC2, 5'-CTTCCTATCCGTCTTGGCAGAC-3', R: 5'-CTCCAGACAGATGGCAATCAGG-3'; CASC9, F: 5'-CTCAGTAGTGGTATGTGCTCAG-3', R: 5'-GGGTACAGTATGTCAGCACAG-3'; miR382-5p, F: 5,-CAAGCATCATGCAGCTCTTC-3, R:5'-TCCCAGAATTTCCAGGCTTA-3'; U6, F: 5'-CAGTGTGAGGTCCTTTCCATCC-3', R: 5'-GCCATAGATGCTTGCGACTGTG-3'; GAPDH, F: 5'-CTCAGTAGTGGTATGTGCTCA G-3', R: 5'-GGGTACAGTATGTCAGCACAG-3'. The PCR thermal cycle parameters were: $95^{\circ}$ for $5 \mathrm{~min}$, followed by three steps: $94^{\circ}$ denaturation for 30 $\mathrm{s}$ and $60^{\circ}$ annealing for $30 \mathrm{~s}$. A total of 45 cycles were carried out. The results were analyzed by $2^{-\Delta \Delta \mathrm{Ct}}$ method.

\section{Western blotting:}

After the protein was extracted, the protein concentration was detected according to the guidance of diquinoline formic acid kit. The extracted protein was added with loading buffer, heated to $95^{\circ}$ for 10 min. The drug loading of each well sample was $30 \mu \mathrm{g}$, adding $10 \%$ polyacrylamide gel to isolate protein. The voltage of gel electrophoresis was controlled at $80 \sim 120 \mathrm{~V}$ and the pressure of wet transport and membrane transfer was controlled at $100 \mathrm{mV}$ for 45 70 min. After polyvinylidene fluoride (PVDF) was transferred, the protein was sealed in $5 \%$ bovine serum protein for $1 \mathrm{~h}$ and then one antibody was added to the membrane and then cultured overnight at $4^{\circ}$. The protein was washed with membrane washing buffer for 3 times, 5 min each time and then the second antibody was added and cultured for $1 \mathrm{~h}$. After washing the membrane three times again, the chemiluminescence reagent developer protein was added. GAPDH was used as the control and Image J software was used to analyze the gray level of the target protein band.

\section{CCK-8 assay:}

HCC 827 and HCC827/G cells in logarithmic growth phase were seeded on 96 well plates, with $10^{4}$ cells in each well that containing $100 \mu 1$ medium. Add 10 $\mu \mathrm{CCK}-8$ solution to each well $1 \mathrm{~h}$ before the test. The culture plates were incubated in the incubator for 1 4 h. The optical density (d) at $450 \mathrm{~nm}$ was measured by microplate to analyze the proliferation activity of NSCLC cells.

\section{Transwell assay:}

The transfected cells were selected as the experimental group and the non-transfected cells as the control group. The cells of each treatment group were digested with trypsin and inoculated into 24 well plate of transwell chamber. Add $100 \mu 1$ cell suspension $\left(2 \times 10^{5} / \mathrm{ml}\right)$ to the upper chamber and add $250 \mu \mathrm{l}$ medium containing $10 \%$ fetal bovine serum to the lower chamber. After incubated for $48 \mathrm{~h}$ in 5 $\% \mathrm{CO}_{2}$ at $37^{\circ}$, the chamber was taken out. The cells in the upper chamber of the microporous membrane were wiped off with cotton swabs and the cells in the upper and lower chambers were carefully washed with phosphate buffered saline (PBS) twice. The cells that invaded and adhered to the microporous membrane of the chamber were fixed with $4 \%$ paraformaldehyde for $15 \mathrm{~min}$ and stained with crystal violet for $15 \mathrm{~min}$. After then, the chamber was washed with PBS, dried and observed under 100 fold inverted microscope.

\section{Cell flow cytometry:}

Flow cytometry was used to detect cell apoptosis. HCC 827 and HCC827/G cells in transfection group and non-transfection group were selected and cultured to logarithmic growth phase. The cells were washed twice with PBS. Mix cells evenly with 500 $\mu \mathrm{L}$ precooling $1 \times$ combined buffer and $5 \mu \mathrm{l}$ AnnexinV-FITC, incubate at room temperature for $15 \mathrm{~min}$. Then, add $2.5 \mu 1$ propidium iodide (PI) staining $5 \mathrm{~min}$ before operation to detect the apoptosis of $\mathrm{HCC} 827$ and $\mathrm{HCC} 827 / \mathrm{G}$ cells.

\section{Dual luciferase reporter assay:}

Firstly, the candidate target molecule miR-382$5 \mathrm{p} 3$ 'UTR of CASC9 or the candidate target genes PDK1 and mTORC2 3'UTR of miR-382-5p were inserted into the downstream of firefly luciferase 
gene. The expression vector pcDNA-enhanced green fluorescent protein (EGFP)-pre-CASC9 and its target gene miR-382-5p verification vector pmirglo-CASC9-miR-382-5p 3'UTR, as well as the expression vector pcDNA-egfp-pre-miR-382-5p and its target gene PDK1 and mTORC2 verification vector pmirglo-miR-382-5p-PDK1-mTORC2 3'UTR were cotransfected into HCC827 cells respectively, and the empty vector was cotransfected with CASC9 or miR-382-5p expression vector. In 24 well plates, $200 \mathrm{ng}$ of pmir glo Mir gene 3'UTR, $600 \mathrm{ng}$ of pcDNA EGFP pre miRNA and $30 \mathrm{pmol} / 1$ of negative control were transfected into each well. $50 \mu \mathrm{l}$ opti minimal essential medium (MEM) I medium diluted $2 \mu$ Lipofectamine ${ }^{\mathrm{TM}} 2000$, incubated at room temperature for $5 \mathrm{~min}$; The DNA to be transfected and diluted Lipofectamine ${ }^{\mathrm{TM}} 2000$ were mixed. After incubation at room temperature for $20 \mathrm{~min}$, the complex was directly added to the cells containing $0.4 \mathrm{ml}$ opti MEM I medium and the culture plate was gently shaken to mix. The cells were cultured in 5 $\% \mathrm{CO}_{2}$ incubator at $37^{\circ}$ for $8 \mathrm{~h} .0 .5 \mathrm{ml}$ of normal DMEM medium containing $10 \%$ fetal bovine serum and no antibiotics was replaced. The cells were cultured in $5 \% \mathrm{CO}_{2}$ incubator at $37^{\circ}$ for $48 \mathrm{~h}$. Luciferase was detected according to the instructions of dual luciferase reporter gene kit. The fluorescence values of firefly and sea cucumber were detected by microplate reader and the fluorescence values of sea cucumber were used as internal reference.

\section{Statistical methods:}

Statistical package for the social sciences (SPSS) 20.0 software was used for statistical analysis, $t$ test was used for comparison between the two groups and Graphpad prism 8 was used to draw relevant pictures of the experimental data. $p<0.05$ or $p<0.01$ meant the difference was statistically significant.

\section{RESULTS AND DISCUSSION}

The expression level of CASC9 in NSCLC was significantly higher than that in normal tissues $(\mathrm{p}<0.01$, fig. 1A). Meanwhile, the expression level of CASC9 in $\mathrm{HCC} 827 / \mathrm{G}$ cells was significantly higher than that in HCC827 cells $(p<0.01$, fig. 1B). The results indicated that the abnormal expression of CASC9 may be related to the occurrence and development of NSCLC and gefitinib resistance.

qRT-PCR showed that si-CASC9 could significantly decrease while pcDNA-CASC9 could significantly increase the CASC9 expression level in $\mathrm{HCC} 827$ and $\mathrm{HCC} 827 / \mathrm{G}$ cells (fig. 2A-fig. 2B, $\mathrm{p}<0.01$, respectively). CCK-8 assay showed that silence of CASC9 could significantly inhibit the proliferation of HCC 827 and $\mathrm{HCC} 827 / \mathrm{G}$ cells, while overexpression of CASC9 could significantly promote the proliferation of HCC 827 and HCC $827 / \mathrm{G}$ cells (fig. $2 \mathrm{C}$-fig. $2 \mathrm{D}, \mathrm{p}<0.05$, respectively). Transwell assay indicated that silencing CASC9 could significantly inhibit the invasion ability of HCC 827 and $\mathrm{HCC} 827 / \mathrm{G}$ cells (fig. 2E, $\mathrm{p}<0.01$ ). Cell flow cytometry showed that si-CASC9 could significantly promote the apoptosis of $\mathrm{HCC} 827$ and HCC 827/G cells (fig. 2F, $\mathrm{p}<0.01)$. These results demonstrated that silencing CASC9 could significantly promote gefitinib induced apoptosis and inhibit cell proliferation and invasion in $\mathrm{HCC} 827$ and $\mathrm{HCC} 827 / \mathrm{G}$ cells.

B

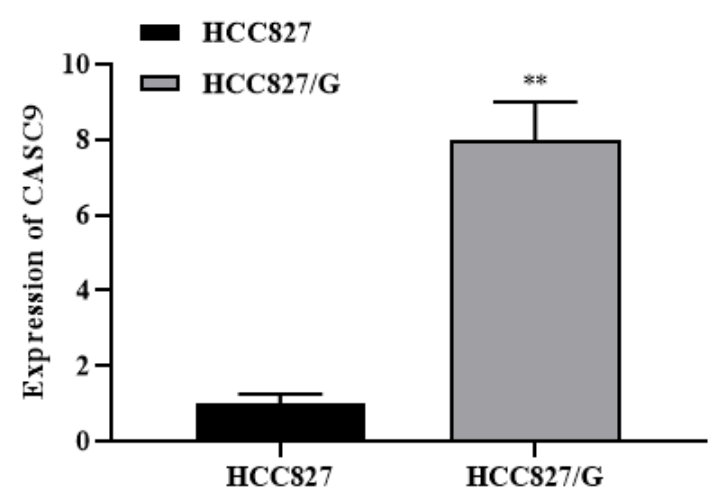

Fig. 1: Detection of CASC9 expression in NSCLC tissues and cells by qRT-PCR, ( $\bullet$ ) Normal tissues, ( $\bullet$ ) Tumor tisues, ( ( ) ) HCC827, ( ロ ) HCC827/G 
www.ijpsonline.com
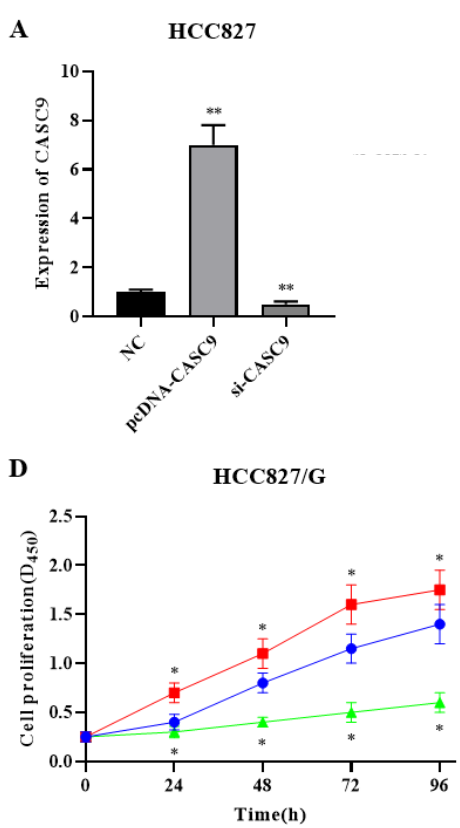

B

$\mathbf{E}$
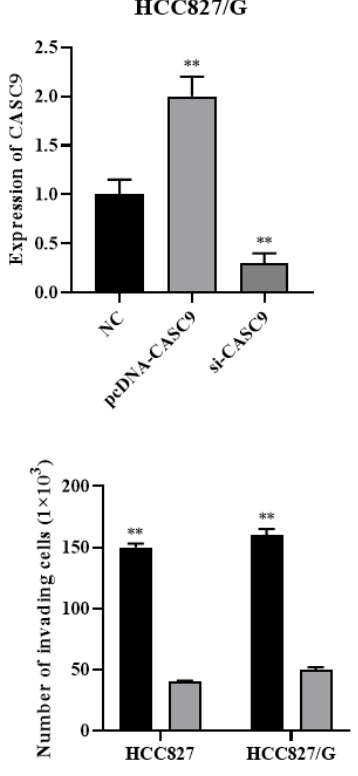

C

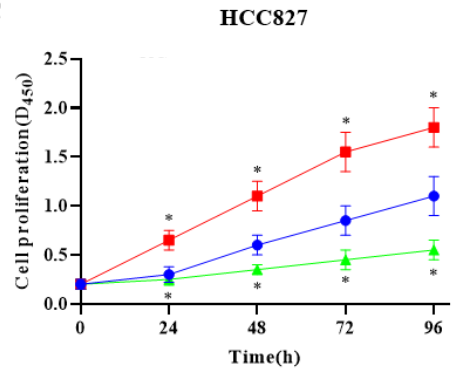

F

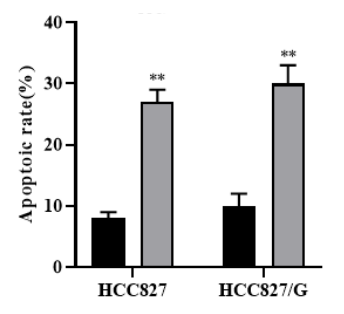

Fig. 2: si-CASC9 promotes proliferation, invasion and inhibits apoptosis of HCC827 and HCC827/G cells, ( $(\square)$ NC, ( $\square$ )

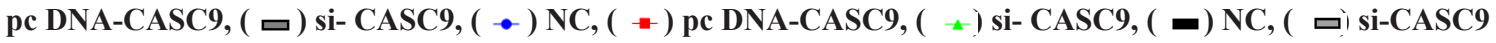

Dual luciferase reporter assay showed that overexpression of miR-382-5p could significantly decrease luciferase activity, while co-transfection of miR-382-5p mimics and pmirglo-CASC9-mut vector with mutation of target site resulted in loss of inhibition effect of miR-382-5p on luciferase activity $(\mathrm{p}<0.01$, fig. 3A). qRT-PCR analysis showed that CASC9 overexpression significantly inhibited the expression of miR-382-5p, while CASC9 knockdown significantly promoted the expression of miR-382$5 \mathrm{p}(\mathrm{p}<0.001$, fig. 3B). These results indicated that CASC9 could negatively regulate the expression of miR-382-5p.

Overexpression of miR-382-5p significantly inhibited the proliferation of $\mathrm{HCC} 827 / \mathrm{G}$ cells, while overexpression of miR-382-5p and CASC9 significantly restored the proliferation of $\mathrm{HCC} 827 / \mathrm{G}$ cells $(p<0.01$, fig. 4A). Overexpression of miR$382-5 p$ significantly inhibited the invasion and promoted the apoptosis of $\mathrm{HCC} 827 / \mathrm{G}$ cells, while overexpression of miR-382-5p and CASC9 simultaneously had no significant effect on the invasion and apoptosis of HCC827/G cells $(p<0.01$, fig. 4B- fig. 4C). These results indicated that CASC9 can promote the proliferation, invasion and inhibit apoptosis of HCC827/G cells by down regulating miR-382-5p.

The bioinformatics database targets can predict that PDK1 and mTORC2 were candidate target genes of
miR-382-5p. Dual luciferase reporter assay showed that miR-382-5p could negatively regulate the expression of PDK1 and mTORC2 ( $\mathrm{p}<0.01$, fig. 5A). Besides, western blot and RT-PCR results showed that overexpression of miR-382-5p significantly inhibit the expression of PDK1 and mTORC2 in HCC827/G cells ( $p<0.01$, fig. 5B-fig. 5C). These results indicated that PDK1 and mTORC2 are the target genes of miR$382-5 p$ and miR-382-5p can negatively regulate the expression of PDK1 and mTORC2.

Western blot and RT-PCR results showed that silencing PDK1 and mTORC2 significantly inhibited the expression of PDK1 and mTORC2, while simultaneously silencing CASC9, PDK1 and $\mathrm{mTORC} 2$ significantly restored the expression of PDK1 and mTORC2 ( $<<0.01$, fig. 6A-fig. 6B). Compared with the control group, si-mTORC2 significantly inhibited cell proliferation and invasion and promoted cell apoptosis. However, silencing the expression of CASC9, PDK1 and mTORC2 at the same time could significantly restore the effect of si-mTORC2 on the proliferation, invasion and apoptosis of $\mathrm{HCC} 827 / \mathrm{G}$ cells ( $<<0.01$, fig. 6C-fig. $6 \mathrm{E})$. These results indicated that $\mathrm{CASC} 9$ up regulates the drug resistance of $\mathrm{HCC} 827 / \mathrm{G}$ cells to gefitinib by down regulating the inhibitory effect of miR-382$5 p$ on PDK1 and mTORC2, thereby promoting the proliferation, invasion and inhibiting apoptosis of HCC $827 / G$ cells. 
$\mathbf{A}$

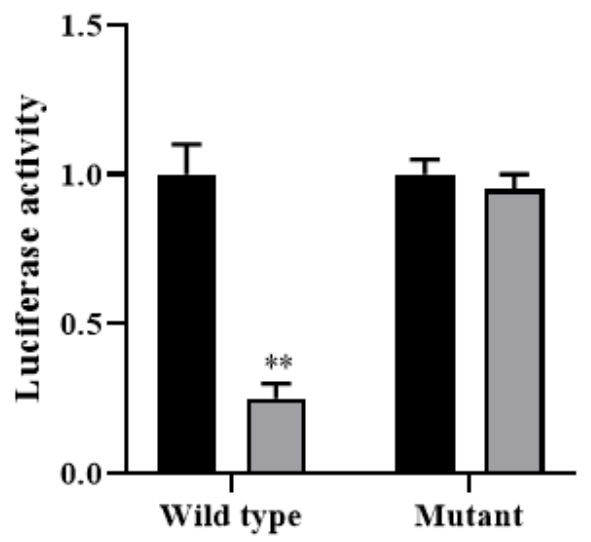

B

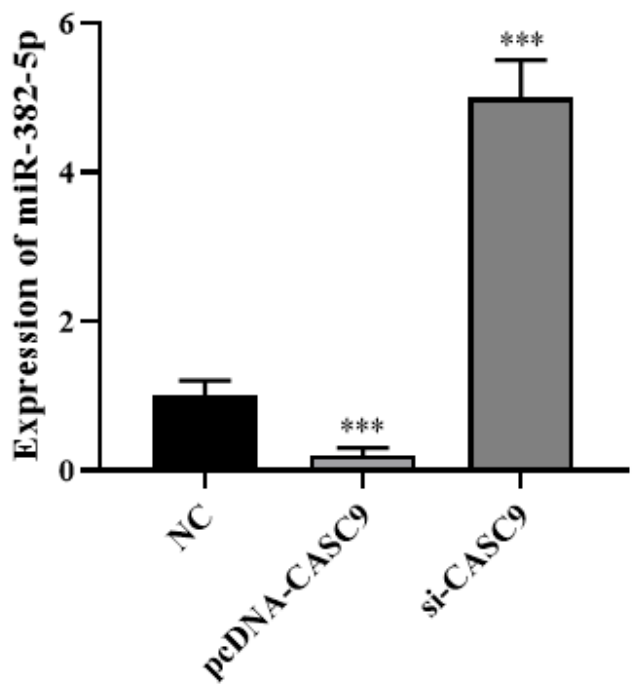

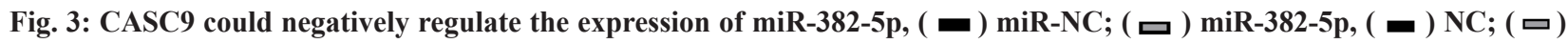
pcDNA-CASC9; ( ロ ) si-CASC9

A

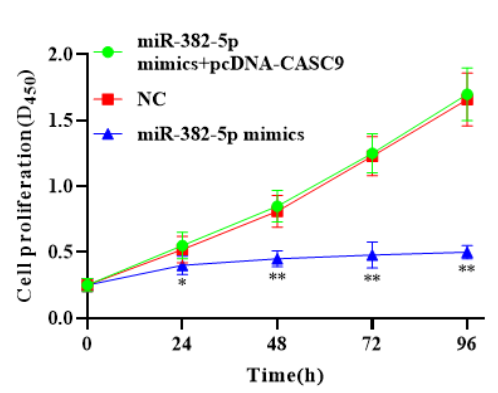

B

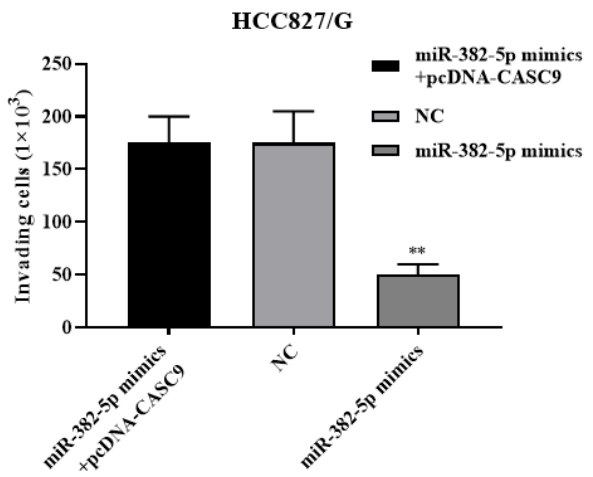

C

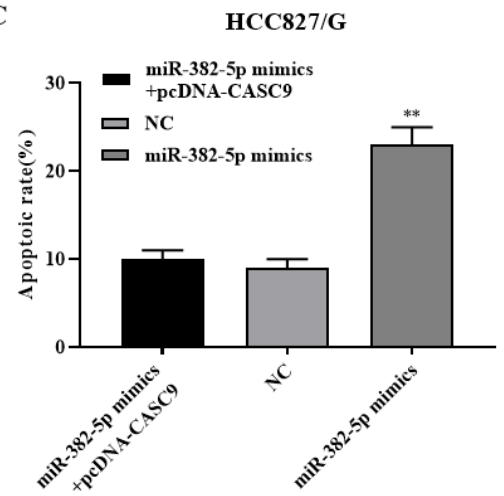

Fig. 4: Effect of overexpression of miR-382-5p on biological behavior of HCC827/G cellsc, $(-\bullet)$ miR-382-5p mimics+pcDNA-CASC9

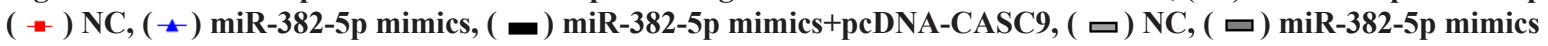

$\mathbf{A}$

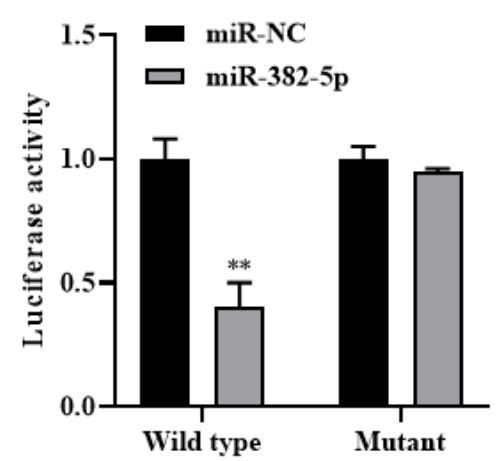

B

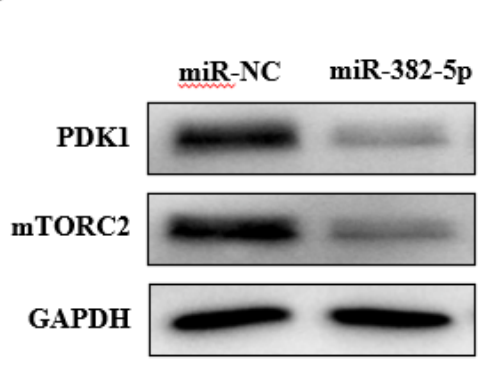

C

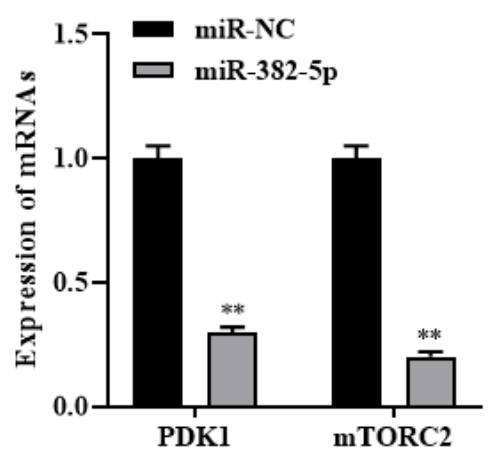

Fig. 5: miR-382-5p regulates the expression of PDK1 and mTORC2, ( $(\square)$ miR-NC, ( $\square$ ) miR-382-5p 

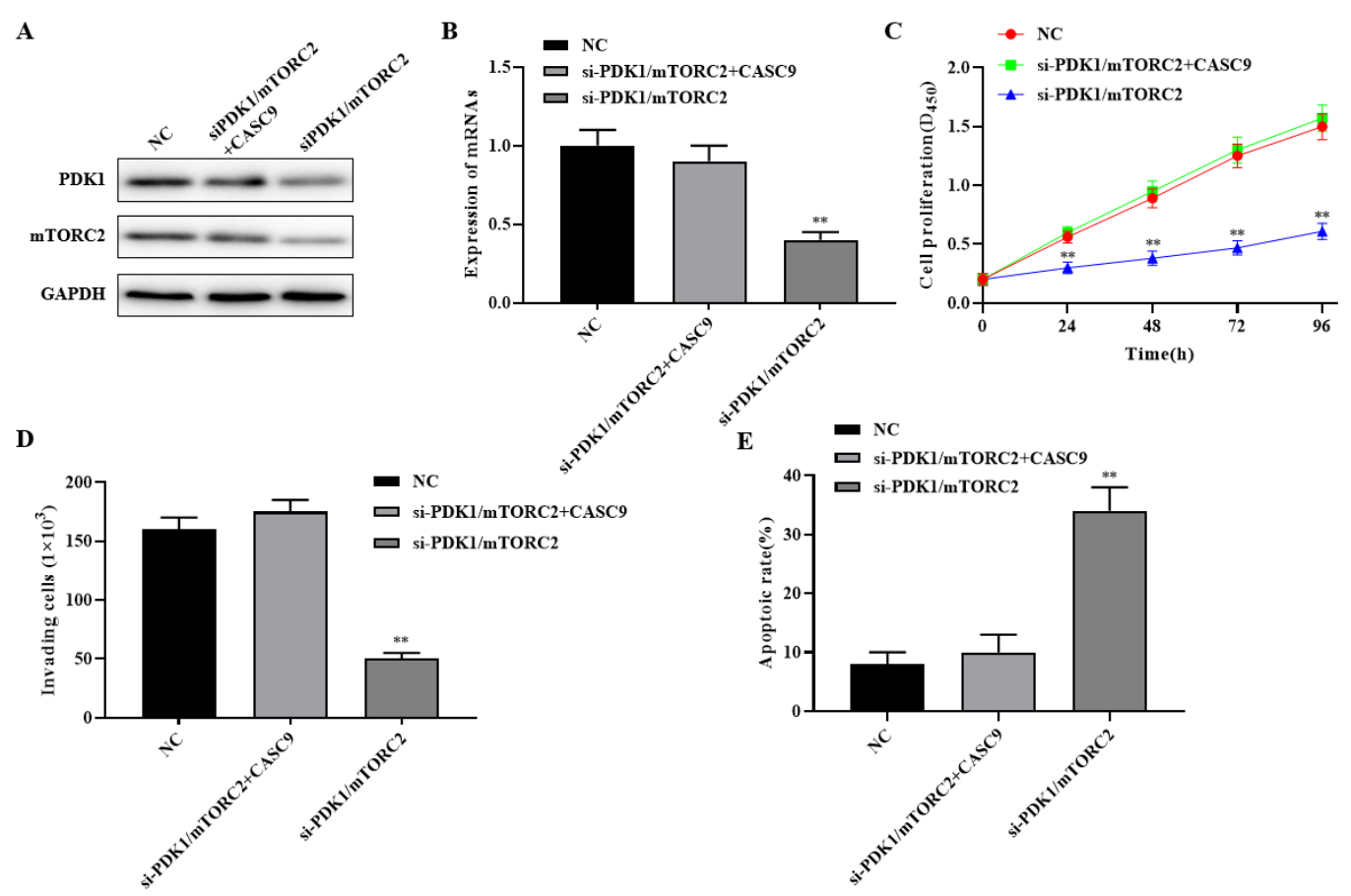

Fig. 6: Casc9 affects biological behavior of HCC827/G cells through miR-382-5p/PDK1/mTORC2 axis, ( $)$ NC, ( 1 ) si-PDK1/mTORC2+CASC9, ( ( ) si-PDK1/mTORC2+CASC9, (-) NC, (-) si-PDK1/mTORC2+CASC9, ( • $)$ si-PDK1/mTORC2

NSCLC accounts for about $80 \%$ to $85 \%$ of lung cancer and most patients have lost the chance of surgical treatment because they are in advanced stage at the time of diagnosis. Chemotherapy is the most important treatment, but the obvious side effects limit the role of chemotherapy in the treatment of advanced NSCLC. Recent studies have proved that ${ }^{[9]}$, due to the tolerance of NSCLC to chemotherapy drugs, the expected curative effect cannot be achieved ${ }^{[10}$, 11]. In recent years, IncRNA has been confirmed to play an important regulatory role in the occurrence and development of tumor and drug resistance ${ }^{[12,13]}$. However, the role of IncRNA in multi-drug resistance of NSCLC remains unclear. Therefore, it is urgent to find the upstream key molecules of the genes related to drug resistance in NSCLC and further study their molecular mechanisms, so as to provide more evidence for the clinical treatment of NSCLC.

In recent years, in addition to structural non-coding RNAs and various small RNAs (miRNAs), researchers have also found a class of lncRNAs with a length of more than 200 nucleotides. LncRNA regulates gene expression in a variety of ways, including epigenetic regulation, transcriptional regulation and post transcriptional regulation. In a word, lncRNAs regulate the occurrence and development of NSCLC, thyroid cancer, colon cancer, liver cancer and other tumors and the process of chemotherapy drug resistance by participating in a variety of biological mechanisms such as $\mathrm{X}$ chromosome silencing, genomic imprinting and DNA damage response ${ }^{[14]}$. Recently, it has been reported that lncRNA metallothionein $1 \mathrm{~J}$ pseudogene (MT1JP) ${ }^{[15]}$, lncRNA Actin filament associated protein 1 (AFAP1) ${ }^{[16]}$, lncRNA small nucleolar RNA host gene 8 (SNHG8) [17] have significant effects on the proliferation, migration and epithelial mesenchymal transition of lung cancer cells, suggesting that lncRNA may be closely related to the occurrence and development of lung cancer. However, the molecular mechanism of lncRNA CASC9 resistance to gefitinib in NSCLC cells has not been reported.

At the same time, recent studies have found that the abnormal regulation of miRNAs is considered to be a key factor in the occurrence and development of many diseases, including NSCLC. For example, miR-382-5p inhibits the proliferation and migration of NSCLC cells by down regulating the expression of Tripartite motif containing 29 (TRIM29) ${ }^{[18]}$. Zhang et al. ${ }^{[19]}$ confirmed that miR-382-5p can be used as an independent prognostic marker for NSCLC. At the same time, some studies have shown that miR-382-5p has significant effects on the proliferation, migration and epithelial mesenchymal transition of lung cancer $^{[20]}$, colorectal cancer ${ }^{[21]}$, pancreatic cancer ${ }^{[22]}$ and liver cancer ${ }^{[23]}$. However, the mechanism of miR$382-5$ p on NSCLC has not been reported. 
According to bioinformatics tools, PDK1 and mTORC2 are predicted to be possible target genes of miR-382-5p. PDK 1 and mTORC2 are members of cell cycle family and their abnormal expression may lead to abnormal proliferation of cells. Studies have found that PDK1 and mTORC2 are abnormally expressed in a variety of tumor tissues, such as cervical cancer, non-small cell lung cancer, non-small cell lung cancer and prostate cancer. Chen et al. ${ }^{[24]}$ reported that miR-615 inhibits the proliferation and invasion of prostate cancer cells by down regulating of PDK1 and mTORC2. In addition, miR-1297 ${ }^{[25]}$, miR-154 ${ }^{[26]}$ and $\mathrm{miR}-497^{[27]}$ have been reported to down regulate PDK1 and mTORC2 to inhibit the proliferation and migration of tumor cells. Therefore, we speculate that miR-382-5p may regulate the proliferation and invasion of NSCLC cells through targeting PDK1 and $\mathrm{mTORC} 2$.

In this study, CASC9 was highly expressed in NSCLC tissues and drug-resistant HCC 827/G cells. Overexpression of CASC9 can significantly promote the proliferation, invasion and inhibit apoptosis of HCC $827 / G$ cells. At the same time, dual luciferase reporter assay confirmed that CASC9 could negatively regulate the expression of miR-382-5p and miR-185-5p could bind to the 3'UTR of PDK1 and $\mathrm{mTORC} 2$ and negatively regulate the expression of PDK1 and mTORC2. Further experiments showed that CASC9 could up regulate the drug resistance of HCC $827 / G$ cells to gefitinib by down regulating the inhibition of miR-382-5p on PDK1 and mTORC2, thereby promoting the proliferation, invasion and inhibiting apoptosis of $\mathrm{HCC} 827 / \mathrm{G}$ cells.

\section{Acknowledgement:}

This work was supported by the Science and Technology Project of Fujian Natural Science Foundation (No. 2018J01204).

\section{Conflicts of interest:}

The authors report no conflicts of interest.

\section{REFERENCES}

1. Roth A, Diederichs S. Long Noncoding Rnas in Lung Cancer. Curr Top Microbiol Immunol 2016;394:57-110.

2. Dai J, Xu LJ, Han GD, Jiang HT, Sun HL, Zhu GT, et al. Downregulation of long non-coding RNA ITGB2-AS1 inhibits osteosarcoma proliferation and metastasis by repressing Wnt/ $\beta$-catenin signalling and predicts favourable prognosis. Artif Cells Nanomed Biotechnol 2018;46(3):S783-90.

3. Wang L, Ma L, Xu F, Zhai W, Dong S, Yin L, et al. Role of long non-coding RNA in drug resistance in non-small cell lung cancer. Thorac Cancer 2018;9(7):761-8.
4. Yang $\mathrm{M}$, Qin Q, Zhu J, Guo Y, Yin T, Wu H, Wang C. Long noncoding RNA ITGB2-AS1 promotes growth and metastasis through miR-4319/RAF1 axis in pancreatic ductal adenocarcinoma. J Cell Physiol 2020.

5. Pietas A, Schlüns K, Marenholz I, Schäfer BW, Heizmann $\mathrm{CW}$, Petersen I. Molecular cloning and characterization of the human S100A14 gene encoding a novel member of the S100 family. Genomics 2002;79(4):513-22.

6. Salmena L, Poliseno L, Tay Y, Kats L, Pandolfi PP. A ceRNA hypothesis: the Rosetta Stone of a hidden RNA language?. Cell 2011;146(3):353-8.

7. Chheang S, Brown K. Lung cancer staging: clinical and radiologic perspectives. Semin Interv Radiol 2013;30(2):99113.

8. Qian J, Ding F, Luo A, Liu Z, Cui Z. Overexpression of S100A14 in human serous ovarian carcinoma. Oncol Lett 2016;11(2):1113-9.

9. Bhan A, Soleimani M, Mandal SS. Long noncoding RNA and cancer: a new paradigm. Cancer Res 2017;77(15):3965-81.

10. Katono K, Sato Y, Kobayashi M, Saito K, Nagashio R, Ryuge S, et al. Clinicopathological significance of S100A14 expression in lung adenocarcinoma. Oncol Res Treat 2017;40(10):594602.

11. Li DY, Chen WJ, Luo L, Wang YK, Shang J, Zhang Y, et al. Prospective lncRNA-miRNA-mRNA regulatory network of long non-coding RNA LINC00968 in non-small cell lung cancer A549 cells: a miRNA microarray and bioinformatics investigation. Int J Mol Med 2017;40(6):1895-906.

12. Liang Y, Chen X, Wu Y, Li J, Zhang S, Wang K, et al. LncRNA CASC9 promotes esophageal squamous cell carcinoma metastasis through upregulating LAMC2 expression by interacting with the CREB-binding protein. Cell Death Differ 2018;25(11):1980-95.

13. Ding F, Wang D, Li XK, Yang L, Liu HY, Cui W, et al. Overexpression of S100A14 contributes to malignant progression and predicts poor prognosis of lung adenocarcinoma. Thorac Cancer 2018;9(7):827-35.

14. Pouyanrad S, Rahgozar S, Ghodousi ES. Dysregulation of miR-335-3p, targeted by NEAT1 and MALAT1 long noncoding RNAs, is associated with poor prognosis in childhood acute lymphoblastic leukemia. Gene 2019;692:35-43.

15. Bray F, Ferlay J, Soerjomataram I, Siegel RL, Torre LA, Jemal A. Global cancer statistics 2018: GLOBOCAN estimates of incidence and mortality worldwide for 36 cancers in 185 countries. CA: Cancer J Clin 2018;68(6):394-424.

16. $\mathrm{Hu} \mathrm{X}, \mathrm{Li}$ Y, Kong D, Hu L, Liu D, Wu J. Long noncoding RNA CASC9 promotes LIN7A expression via miR-758-3p to facilitate the malignancy of ovarian cancer. J Cell Physiol 2019;234(7):10800-8.

17. Yu L, Hao Y, Xu C, Zhu G, Cai Y. LINC00969 promotes the degeneration of intervertebral disk by sponging miR-335-3p and regulating NLRP3 inflammasome activation. IUBMB life 2019;71(5):611-8.

18. Zhao X, Tang DY, Zuo X, Zhang TD, Wang C. Identification of IncRNA-miRNA-mRNA regulatory network associated with epithelial ovarian cancer cisplatin-resistant. J Cell Physiol 2019;234(11):19886-94.

19. Zhang J, Wang Q, Quan Z. Long non-coding RNA CASC9 enhances breast cancer progression by promoting metastasis through the meditation of miR-215/TWIST2 signaling associated with TGF- $\beta$ expression. Biochem Biophys Res Commun 2019;515(4):644-50. 
20. Luo K, Geng J, Zhang Q, Xu Y, Zhou X, Huang Z, et al. LncRNA CASC9 interacts with CPSF3 to regulate TGF- $\beta$ signaling in colorectal cancer. J Exp Clin Cancer Res 2019;38(1):1-6.

21. Li M, Chen Y, Zhu J, Gao Z, Wang T, Zhou P. Long noncoding RNA CASC15 predicts unfavorable prognosis and exerts oncogenic functions in non-small cell lung cancer. Am J Transl Res 2019;11(7):4303-14.

22. Jiao P, Hou J, Yao M, Wu J, Ren G. SNHG14 silencing suppresses the progression and promotes cisplatin sensitivity in non-small cell lung cancer. Biomed Pharmacother 2019;117:109164.

23. Ren Y, Zhang H, Jiang P. MicroRNA-382 inhibits cell growth and migration in colorectal cancer by targeting SP1. Biol Res 2018;51(1):1-8.

24. Chen L, Nan A, Zhang N, Jia Y, Li X, Ling Y, et al. Circular RNA 100146 functions as an oncogene through direct binding to miR-361-3p and miR-615-5p in non-small cell lung cancer. Mol Cancer 2019;18(1):1-8.

25. Wang $Y$, Xue J, Kuang $H$, Zhou $X$, Liao L, Yin $F$. microRNA-1297 inhibits the growth and metastasis of colorectal cancer by suppressing cyclin D2 expression. DNA Cell Biol 2017;36(11):991-9.
26. Zhu C, Shao P, Bao M, Li P, Zhou H, Cai H, et al. miR-154 inhibits prostate cancer cell proliferation by targeting CCND2. Urol Oncol 2014;32(1):31-e9.

27. Zhong H, Yang J, Zhang B, Wang X, Pei L, Zhang L, et al. LncRNA GACAT3 predicts poor prognosis and promotes cell proliferation in breast cancer through regulation of miR-497/ CCND2. Cancer Biomark 2018;22(4):787-97.

This is an open access article distributed under the terms of the Creative Commons Attribution-NonCommercial-ShareAlike 3.0 License, which allows others to remix, tweak, and build upon the work non-commercially, as long as the author is credited and the new creations are licensed under the identical terms

This article was originally published in a special issue,

"Therapeutic Perspectives in Biomedical Research and Pharmaceutical Sciences and their Nursing Methods"

Indian J Pharm Sci 2021:83(4)Spl issue "144-152" 